\section{HPV-positives OPC: Cetuximab zusätzlich zur Strahlentherapie verspricht Vorteile}

Immer häufiger wird bei Patienten mit oropharyngealen Karzinomen (OPC) eine Infektion mit humanem Papillomavirus (HPV) nachgewiesen. Retrospektiv wurde nun nach Unterschieden bei Überlebensparametern von HPV-positiven und -negativen Patienten gesucht.

$B^{c}$ ei $45-90 \%$ der Patienten mit neu diagnostiziertem oropharyngealem Karzinom (OPC) lässt sich eine Infektion mit humanem Papillomavirus (HPV) bzw. eine p16-Expression nachweisen. Die HPV-positiven Patienten sind meist jünger als die „klassischen“ Fälle mit Tabak- und/oder Alkohol-assoziiertem OPC und haben weniger Komorbiditäten. Entsprechend gut ist ihr Überleben unter Chemoradiotherapie - allerdings mit dem Risiko später Toxizitäten. Der gegen EGFR („epidermal growth factor receptor") gerichtete Antikörper Cetuximab ist eine weitere Therapieoption bei dieser Entität, meist in Kombination mit Strahlen- und/oder Chemotherapie gegeben. Inwieweit HPV-positive Patienten mit lokoregionär fortgeschrittenem OPC im Vergleich zu HPV-negativen Patienten von der Gabe von Cetuximab zusätzlich zur Strahlentherapie (RT) profitieren, wurde in einer retrospektiven Subgruppenanalyse der Phase-III-Studie IMCL-9815 mit 182 OPCPatienten mit bekanntem p16-Status untersucht.

$41 \%$ der Patienten waren p16-positiv. Unter RT wie auch unter RT plus Cetuximab zeigten diese Patienten ein im Vergleich zu den HPV-negativen Patienten signifikant längeres Gesamtüberle- ben (OS) (Hazard Ratio [HR] 0,40; $95 \%$-Konfidenzintervall [95\%-KI] 0,210,74 bzw. HR 0,16; $95 \%$-KI 0,07-0,36). Cetuximab verbesserte lokoregionäre Kontrolle, OS und progressionsfreies Überleben bei p16-positiven wie auch p16-negativen Patienten. Vergleichbare Ergebnisse zeigten sich bei Patienten mit p16-positivem/HPV-positivem $(\mathrm{n}=49)$ und mit p16-positivem/HPV-negativem OPC $(\mathrm{n}=14)$.

Fazit: Eine Subgruppenanalyse ergab eine vergleichbare Verbesserung des Therapieerfolgs durch die Behandlung mit RT plus Cetuximab bei Patienten mit p16-/HPV-positivem OPC und bei Patienten ohne nachweisbare Virusinfektion.

Barbara Kreutzkamp

Rosenthal DI et al. Association of human papillomavirus and p16 status with outcomes in the IMCL-9815 phase III registration trial for patients with locoregionally advanced oropharyngeal squamous cell carcinoma of the head and neck treated with radiotherapy with or without cetuximab. J Clin Oncol. 2016;34(12):1300-8.

\title{
Optimierung der Nachsorge bei HPV-positiven Oropharynxtumoren
}

Oropharyngeale Plattenepithelkarzinome (OPSCC) mit HPV-Nachweis zeigen einen günstigeren Verlauf als HPV-negative Tumoren. Eine retrospektive Untersuchung mit Blick auf Rezidive oder Spättoxizität nach der Therapie legt nahe, dass eine Deeskalation im Nachsorgemanagement möglich ist.

$\mathrm{n}$ die retrospektive Analyse gingen 232 Patienten ein, die zwischen 2005 und 2013 wegen eines $\operatorname{HPV}($ „human papilloma virus")-positiven $\left(\mathrm{HPV}^{+}\right)$OPSCC eine Radio(chemo-)therapie erhalten hatten. Die Patienten wurden im 1. Jahr nach Therapieende alle 3 Monate nachuntersucht, im 2. Jahr alle 4 und in den Jahren 3-5 alle 6 Monate. Prüfparameter waren die Manifestation von Spättoxizität $(\geq$ Grad 3), das Auftreten lokoregionärer Rezidive oder distaler Metastasen sowie das Gesamtüberleben (OS). Es wurde eine Stratifizierung in 2 Risikogruppen (niedrig oder intermediär) entsprechend der RTOG-0129-Studie (RTOG; Radiation Therapy Oncology Group) vorgenommen. Die Niedrigrisiko-Gruppe $(\mathrm{n}=162 ; 70 \%)$ war definiert als Patienten mit nicht me- tastasiertem, $\mathrm{HPV}^{+}$OPSCC mit einer Raucheranamnese von $\leq 10$ Packungsjahren bzw. > 10 Packungsjahren und einem N-Stadium von N0-2a, die Gruppe mit intermediärem Risiko $(\mathrm{n}=70 ; 30 \%)$ als Patienten mit nicht metastasiertem, $\mathrm{HPV}^{+}$ OPSCC mit einer Raucheranamnese von $>10$ Packungsjahren und dem N-Stadium N2b-N3.

Die große Mehrheit der Patienten (196; 85\%) erhielt parallel zur Radioeine Chemotherapie (überwiegend platinbasiert, $15 \%$ mit Cetuximab). Nach 3 Jahren betrug die lokoregionäre Kontrollrate $94 \%$, die Fernmetastasen-freie Rate $91 \%$. Die Rate für das 3-Jahres-OS belief sich auf $91 \%$. Eine Spättoxizität zeigte sich bei 21 Patienten (9\%). Insgesamt traten $64 \%$ der Toxizitätsereignis- se und Rezidive innerhalb der ersten 6 Monate des Follow-up auf. Bei jedem der späteren Nachsorgetermine betrug die Inzidenz solcher Ereignisse weniger als $2 \%$. Nur 4 Patienten erlebten ein erstes Ereignis später als 2 Jahre nach Ende der Therapie. In der multivariaten Analyse war ein Stadium N3 (intermediäres Risiko) mit einem höheren Risiko für Fernmetastasen verbunden.

Fazit: Das $\mathrm{HPV}^{+}$OPSCC hat nach Therapie ein niedriges Risiko für Rezidive/Metastasen oder Spättoxizität. Etwa 2 von 3 Ereignissen treten in den ersten 6 Monaten des Follow-up auf. Es könnte somit vernünftig sein, die Nachsorgefrequenz $z u$ reduzieren $-z$. B. alle 3 Monate im ersten Halbjahr, alle 6 Monate in den ersten 2 Jahren und danach jährlich.

Brigitte Schalhorn

Frakes JM et al. Determining optimal follow-up in the management of human papillomavirus-positive oropharyngeal cancer. Cancer. 2016;122(4):634-41. 